\title{
Effects and Applications of Music Therapy on Psychological Health: A Review
}

\author{
Junrui Huang ${ }^{1 *}$, Xiaoqing $\mathrm{Li}^{2}$ \\ ${ }^{1}$ AP Department Guangdong Country Garden School Foshan 528000 China \\ ${ }^{2}$ Guangzhou University Affiliated High School Guangzhou 510000 China \\ These authors contributed equally. \\ *Corresponding author. Email: guanghua.ren@gecacdemy.cn
}

\begin{abstract}
Throughout its long history, music has played an indispensable role in our life. Listening to music enables us to relax and calm down, and composing and playing music have been good ways to express our feelings and emotions. Music therapy has been set up to cure more people systematically. In this study, we reviewed the history of music therapy and have focused on several aspects of our psychological health that have been influenced by it. Several treatment therapies related to music therapy have been discussed. In conclusion, music therapy has brought a tremendous impact on people's psychological health, enabled patients to suffer less pain, anxiety, and feeling stressful and thus improves our emotional states, bringing psychological health to the patients.
\end{abstract}

Keywords: music therapy, psychological health, emotion, stress, humanistic therapy

\section{INTRODUCTION}

To find the origin of music therapy, we should date back to ancient Greece. Many famous philosophers in ancient Greece like Plato and Aristotle have set up various viewpoints about music therapy. But it was not until 1944 did music therapy officially became a subject in America. Since then, music therapy has started to develop worldwide and gradually made progress [1]. Music therapy is defined as a curing process based on the relationship between therapists and patients, which contains essential factors like systematic procedures and rigorous assessments. The concept of music therapy is mainly similar to psychotherapy; the only difference between them is the way they treat patients: music therapy's method is music, and psychotherapy primarily uses communication [2]. Over the years, music therapy has been adopted for various disorders, including ASD, PTSD, and Depression.

The relation between music and humans is inseparable. From baroque, classic, romantic to modern, the story of music seems to develop along with humans, becoming a characteristic of the human condition from millennia [3]- music brings people together [4]. During the evolutionary process, our ancestors, the early hominids, were able to emit sounds of variable pitch, showing that music must have predated speech in the simplest level [5]. Nowadays, many people use it for exploring and managing their moods and emotions [6]. Therefore, music's therapeutic effects are becoming more influential, leading researchers to further discuss its impacts on various sides, such as applying music to treat psychological disorders [6] [7]. Moreover, the studies about music in the neuroscience field have also attracted many researchers. Thus, the influential characteristic of music, both in the evolutionary process of the human and scientific fields, brought significant impacts to humans.

However, as putting the attention the music therapy in China, even though music therapy has rising popularity, its development is still lagging off in the academic field and fostering therapists; also, even the inserve educators and therapists need to enhance their skills further [8]. The purpose of this review is to make the frame of what music therapy has contributed to our psychological health clearer and thus enable us to do future research better and enhance the utilization of music therapy. It would be an excellent treating tool for many aspects because it is safe, cheap, and convenient. In this review, we chose several prospects of psychological health that music therapy has a significant influence on and showed how massive the impacts are by looking over several related types of research. 


\section{EFFECTS OF MUSIC THERAPY ON PSYCHOLOGICAL HEALTH}

\subsection{Emotion}

People use various ways to convey their emotional state: language, written words, facial and body expressions, dactylology, etc. Among other sensory systems, auditory signals, voice, for example, are also major approaches to communicating emotion. Music tights with our brain and our auditory system, emphasizing the importance of the right hemisphere in our brain. The impairments of the brain lead to the impairments of appreciation in pitch, tone, and rhythm [9], which are elements related to music; the right hemisphere will initially be activated when listening to music in emotional related experience [10]; listening to music and voice evoke the activity in the limbic system, which is the core structure of emotional processing [11] [12].

The emotions conveyed from voices may include positive (happiness, excitement) and negative emotion (Depression, anger). Many recent studies have shown that music helps arouse humans' positive emotions, leading to psychological well-being. For example, Stefan Koelsch suggested that "music therapy has beneficial effects on the psychological and physiological health of individuals" [11]. Furthermore, in a case study about a patient with epilepsy, music-induced the event-related potential (ERP) to change in the brain, leading to pleasure; also, the ERP results emphasized the implication in the emotional judgment of pleasant music [13]. Thus, music therapy may help to bring positive emotion.

\subsection{Stress}

Stress now appears in various aspects of our daily life: family, work, study, etc. It helps our survival, which regulates our internal physiological states (body temperature, heart rate, etc.), to maintain homeostasis inside our body, allowing us to accommodate everyday situations. However, exposure to stress regularly and repeatedly, in the long run, will also affect people's immune systems, cardiovascular and metabolic systems, and brain function [14].

Recent studies have shown that music is related to stress-reduction effects and increases in well-being. Firstly, Linnemann [15] proposed a study protocol about how music listening affected psychobiological stress in daily life, setting up a more natural situation for participants to listen to music in their daily routine. The conclusion was that a stress-reducing effect of music listening was found, depending on the characteristics of the situation and the music [15]. Secondly, listening to music helped to improve the experience, enhance treatment motivations or stress-related outcomes [16] [17]. For patients who were stressed when undergoing surgeries (cardiac CT), the use of relaxation of music did lead to improved patient experience [17]. In the research about female patients with methamphetamine use disorder, the researchers adopted Group Music Therapy (GMT) and effectively enhanced patients' treatment motivation [16].

\subsection{Anxiety}

Anxiety causes by various aspects of our daily life, and it has many different types. For example, there is Generalized Anxiety Disorder, which worries about different things in our lives; there is a phobia, a specific fear or anxiety of certain events or objects, etc. Severe anxiety disorders have huge negative events on our life.

To relieve anxiety, people tried different methods. One of them was music therapy. In 2015, research on music therapy's influence on thoracic surgery patients was set. Participants were placed in a serene environment and were allowed to control the volume of music by themselves. The therapists told them to relax and focus on the music during the process. The results indicate that participants have gained better self-being and become more peaceful than before [19]. In 2016, a group of Chinese researchers did a study on how music therapy affects prisoners. They randomly assigned the prisoners into different groups and provided the objects with treating methods like music imagery, improvisation, and songwriting. After comparing the data from the control group and experimental group, the result showed that music therapy has a relatively large impact on prisoners' anxiety. In 2020, some therapists tried to adopt music therapy in relieving the anxiety of surgical patients. They provided patients with individualized music therapy and utilized different kinds of treatments according to the patients' situations. After 12 weeks of the experiment, the results showed that music therapy reduced the anxiety of those surgical patients [20].

\subsection{Pain}

Pain exists everywhere in our life. It can narrow down to a scan on our body, and if severe enough, to a pain brought by a disease like cancer that may lead us to death. Also, some pains are generated psychologically, which have nothing to do with physical health. Needless to say, people have been concerned about ways to relieve pain for a long time.

Recent studies showed that music therapy had a significant impact on pain reduction. In 2018, for 30 days, patients with the end-stage live disease would listen to music they liked for three days, 30 minutes per day. By comparing the patients' pain level 15 minutes before music intervention and 30 minutes after the intervention, the researchers gained the result that music therapy reduced $10 \%$ of the pain of the patients [21]. In 2020, a group of music therapists provided one-to-one music 
therapy for the surgical patients enrolled. The result proved that music therapy was effective on surgical patients and could be utilized to reduce the usage of narcotics [20]. In 2021, music therapy was confirmed to have positive impacts on relieving the pain of patients with small-cell lung cancer by conducting six steps including improvisation, music imagery, and so on [22].

\section{THERAPY}

\subsection{Behavioral Therapy}

In behavioral approaches, token economy, through reinforcing the desired behavior by using tokens as rewards and weakening an undesired behavior by offering punishment, could achieve a change in behavior [23]. This method adopted operant conditioning theory, based on the work of B.F. Skinner: "behavior which is reinforced tends to be repeated (i.e., strengthened); behavior which is not reinforced tends to die out-or be extinguished (i.e., weakened)." [24] After combining token economy with music therapy, music can be used as a reinforcer in behavior modification, particularly contingent music and time-out form music [25]. Therefore, therapists could eventually modify clients' behaviors by combining behavioral approaches with music therapy.

\subsection{Humanistic Therapy}

In humanistic therapy, therapists emphasized the inner power of humanity and focused on individuals' capacity to make their own choices, and sought to foster personal growth through direct experience [26] [27]. Client-centered therapy helped therapists build a relationship with the clients, giving them the sense of being understood, cared for, and listened without any judgment. In this way, clients were able to release emotion and actualize themselves in a good way. Though Music therapy has not been used with humanistic approaches directly, therapists could use music to create a good relationship, and also through the use of music, "to reflect both musically and verbally the client's improvisations." [25]

\subsection{Psychodynamic Therapy}

Psychodynamic theories include Sigmund Freud, Carl Jung, Alfred Adler, and other theorists who proposed theories that focused on the unconscious force, motivation and drive, individual development, and the explanation of unconsciousness. Psychodynamic therapists were not only aiming to modify clients' personalities but also seeking to let clients realize the influence of significant events that happened during their growth. Additionally, therapists wanted to help solve the conflicts in unconsciousness [2] and help clients achieve an understanding of themselves. Music could arouse listeners' emotions, which allowed people to feel peace and calm [28]. Consequently, when progressing individual therapy, music could give a sense of security, and became an excellent medium to build a relationship between clients and therapists, and also helped clients to let off some emotion [2]. Besides, adopting music therapy enabled therapists to open clients' inner hearts and helped modify behavior and express feelings in a non-verbal way.

\subsection{Application}

\subsubsection{ASD}

ASD is a type of neurological disorder. Patients will own a fixed behavior pattern, lack of interest, and social contact. Therapists could encourage patients to sing, do improvisation, and play instruments by using music therapy. These activities were recognized to effectively improve patients' attention by following the tempo, imitating the movements, and finally, synchronizing the tempo.

"A positive effect of educational music therapy on patients with ASD was reported in most controlled studies $(6 / 7)$, particularly in terms of speech production; a positive effect of improvisational music therapy was reported in most controlled studies (6/8), particularly in terms of social functioning." [29] For the reason that music therapy has the function of influencing emotions. In another report on children with ASD, researchers adopted experiments about music therapy for children with ASD after searching and analyzing the various database. The result was that music therapy promoted the improvement of ASD children's initial skills like verbal communication, behavior modeling, and the abilities of social and emotional interactions [30].

\subsubsection{Depression}

Depression is also called a depressive disorder, and it is the primary type of mood disorder. The main symptoms of Depression are frequent and consistent feeling down, along with pessimism and hopelessness, interest loss, and even suicidal behavior. Grievous Depression influences people's physical health and everyday life, and its control method is usually medical treatment. Until recent years, investigations on music therapy have become mature gradually, and it was able to open a new field in the treatment of Depression.

Music therapy can be divided into active music therapy and passive music therapy. In active music therapy, patients are encouraged to create their music to improve their mental state. On the other hand, passive music therapy mainly helps patients reach a better mental state by letting them listen, experience, and appreciate music. Many pieces of research have proved the effectiveness of music therapy. Erkkila and his fellows 
put 79 patients with Depression into research and divided them into the control group and experimental group. The control group used regular treatment, and the experimental group added music therapy based on regular treatment. The result showed that the experimental group showed significantly lower depressed and anxious levels than the control group [31].

\subsubsection{PTSD}

Post-traumatic stress disorder (PTSD) is a mental disturbance left by extraordinary events patients have undergone. When patients encounter scenarios or sound similar to previous traumas, they will show specific reaction like fear and escape. During the treating process, therapists first established trust with patients through music singing games. Next, therapists led patients into deep relaxation by playing suitable music to them. Third, therapists led patients back from creative imagination and prompted them to relieve their emotions and thus ease their reactions to their sensitive scenes. After several groups of control experiments, the results showed that compared to patients in the control group, patients in the experimental group had a more obvious reduction in relative symptoms, and they also showed higher satisfaction. As a result, music therapy effectively calmed patients down and thus had reliable clinical effects and high utilization values [32].

\section{DISCUSSION AND CONCLUSION}

As we can see in the previous sections, music therapy was able to generate significant positive effects on our psychological health, including alleviating anxiety, improving our emotional states, relieving our stress, and reducing our pain. Music therapy has been conducted on curing psychological disorders like ASD, Depression, and PTSD. It contributed to our better mental state along with other therapies such as behavioral therapy, humanistic therapy, and psychodynamic therapy.

Although music therapy has made outstanding contributions to our psychological health, it could not cure us if the organic disease were in our bodies. Music therapy can only serve as subsidiarity in the curing process of physical illness [33]. Based on various researches throughout the history of music therapy, it is likely that music therapy can stand out independently in the future.

In some research about music and emotional arousal, the experimenters also needed to consider the music background of the subjects when doing experiments about music, in that many research set up different standards about the questionnaires, which affected the comparison of dividing groups and doing data analysis. Besides, the preference of different music, such as pop music or pure music, needs to be examed cautiously [34].
Besides, the cultural background between Eastern and Western countries may influence the use of music therapy to a large extend. For example, in Wu's paper about the development of music therapy in China in the past 20 years, the application of music therapy at the beginning even led to an increase in tension between clients and their families [35].

For music therapy in the whole picture, there are several directions for future research. Initially, researchers may focus on the psychological effects of different styles of music. The music used for the experiment could be various in the genre, such as jazz, classical, and pop. However, the therapeutic effects of different genres do not get enough attention. Secondly, for music therapy in China, even though there are signs of a combination of traditional Chinese treatment and music therapy, Chinese researchers should find a suitable approach for patients in China. As mentioned above, the western approach may not fit into Chinese society due to the difference in cultural background.

To conclude, music therapy has brought a tremendous impact on people's mental health, enabled patients to suffer less pain, anxiety, and feeling stressful and thus improves our emotional states, bringing psychological health to the patients. Combining with several other theories mentioned, music therapy has been adopted to cure patients with psychological disorders, like Depression, ASD, and PTSD, which we have mentioned. The development of music therapy is in a rising stage in China.

\section{REFERENCES}

[1] Thaut M H. Music as therapy in early history[J]. Progress in Brain Research, 2015, 217(3):143-158.

[2] Kaiyuan Pu. The Theorem of Music Therapy[J]. Medical Science and Philosophy, 1991, 012(001):39-40.

[3] Welch G F, Biasutti M, Macritchie J, et al. Editorial: The Impact of Music on Human Development and Well-Being[J]. Frontiers in Psychology, 2020, 11:1246.

[4] Frontiers. "The story of music is the story of humans: Where did music come from? Recent article discusses how music arose and developed." ScienceDaily, 2017.

[5] Jeremy Montagu. How Music and Instruments Began: A Brief Overview of the Origin and Entire Development of Music, from Its Earliest Stages. Frontiers in Sociology, 2017; 2 DOI: 10.3389/fsoc.2017.00008

[6] Mcferran KS, Hense C, Koike A, et al. Intentional music use to reduce psychological distress in adolescents accessing primary mental health care[J]. 
Clinical Child Psychology \& Psychiatry, 2018:1359104518767231.

[7] Maratos AS, Gold C, Xu W, et al. Music therapy for depression[J]. Cochrane Database Syst Rev, 2008, 108(1):CD004517.

[8] Lu Yue, Peng Yuntong. Music therapy in China[J]. Journal of Exceptional People, Vol.1, No. 6: 83- 88.

[9] Lauren S, Katharina V K, Warren J D, et al. Music and the brain: disorders of musical listening[J]. Brain, 2006, 129(Pt 10):2533-2553.

[10] Blood A J, Zatorre R J, Bermudez P, et al. Emotional responses to pleasant and unpleasant music correlate with activity in paralimbic brain regions.[J]. Nature Neuroscience, 1999, 2(4):382-387.

[11] Koelsch S. A neuroscientific perspective on music therapy.[J]. Annals of the New York Academy of Sciences, 2010, 1169:374-384.

[12] S Frühholz, Trost W, Grandjean D. The role of the medial temporal limbic system in processing emotions in voice and music[J]. Progress in Neurobiology, 2014, 123:1-17.

[13] D Dellacherie, Pfeuty M, D Hasboun, et al. The birth of musical emotion: a depth electrode case study in a human subject with epilepsy.[J]. Ann N Y Acad, 2010, 1169:336-341.

[14] Mcewen BS. Protective and damaging effects of stress mediators.[J]. N Engl J Med, 1998, 338:171179.

[15] Linnemann A, Strahler J, Nater U M. Assessing the Effects of Music Listening on Psychobiological Stress in Daily Life[J]. Journal of Visualized Experiments, 2017(120).

[16] Wu Q, Chen T, Wang Z, et al. Effectiveness of music therapy on improving treatment motivation and emotion in female patients with methamphetamine use disorder: A randomized controlled trial[J]. Substance Abuse, 2019(1):1-8.

[17] Witte M D, Pinho S, Stams G J, et al. Music therapy for stress reduction: a systematic review and metaanalysis[J]. Health Psychology Review, 2020(1).

[18] Ng M Y, Karimzad Y, Menezes R J, et al. Randomized controlled trial of relaxation music to reduce heart rate in patients undergoing cardiac CT[J]. European Radiology, 2016, 26(10):1-8.

[19] Yang L, Petrini M A . Effects of music therapy on pain, anxiety, and vital signs in patients after thoracic surgery[J]. Complementary therapies in medicine, 2015, 23(5):714-718.
[20] Bojorquez G R, Jackson K E, Andrews A K. Music Therapy for Surgical Patients: Approach for Managing Pain and Anxiety[J]. Critical care nursing quarterly, 2020, 43(1):81-85.

[21] Slater, Larry, Navarra, et al. Music Therapy and Pain Management in Patients with End-Stage Liver Disease[J]. Nursing Research, 2017, 66(2): E63E64.

[22] Tang H, Chen L, Wang Y, et al. The efficacy of music therapy to relieve pain, anxiety, and promote sleep quality, in patients with small-cell lung cancer receiving platinum-based chemotherapy[J]. Supportive Care in Cancer, 2021.

[23] American Psychology Association. Token economy. APA Dictionary of Psychology. Retrieved August 17, 2021.

[24] Saul McLeod. "What is Operant Conditioning and How Does It Work?".SimplePsychology, 2018.https://www.simplypsychology.org/operantconditioning.html

[25] Barbara W . The Relationship Between Music Therapy and Theories of Psychotherapy[J]. Music Therapy, 1981(1):9-16.

[26] American Psychology Association. Humanistic psychology. APA Dictionary of Psychology. Retrieved August 17, 2021.

[27] American Psychology Association. Humanistic Therapy. APA Dictionary of PsychologyRetrieved August 17, 2021.

[28] Shahram Heshmat. Music, Emotion, and WellBeing. Psychology Today, 2019.

[29] Mayer-Benarous H, Benarous X, Vonthron F, et al. Music Therapy for Children With Autistic Spectrum Disorder and/or Other Neurodevelopmental Disorders: A Systematic Review $[\mathrm{J}]$. Frontiers in Psychiatry, 2021, 12:643234.

[30] Geretsegger M, Elefant C , Karin A Mössler, et al. Music therapy for people with autism spectrum disorder.[J]. Cochrane Database of Systematic Reviews, 2014, 6(6): CD004381.

[31] Ping $\mathrm{Xu}$, Lijing, Zhao, Yingying Chen, etc. Development of music therapy in recovering of depression patients[j].Chinese Journal of Clinical Health Care, 2020,023(003):316-320.

[32] Aineng Tang, Kaiwen Zou, Xiaona He,etc. Application of music therapy on spinal cord patients with Post-traumatic Stress Disorder (PTSD)[J]. Wisdom Health, 2021,7(12):57-59.

[33] Xiaolian Xiao. Application and development of music therapy $[\mathrm{J}]$. Exploration on Medical Education 
2005 Volume 4, Issue 3, pages 166-167,184, ISTIC, 2005.

[34] Kreutz G, Ott U, D Teichmann, et al. Using music to induce emotions: Influences of musical preference and absorption[J]. Psychology of Music, 2007, 36(1):101-126.

[35] Yue Wu, MM, MT-BC, The Development of Music Therapy in Mainland China[J]. Music Therapy Perspectives, 2019,37(1):84-92. 